\title{
NEW CAD MODEL OF THE MICROSTRIP INTERDIGITAL CAPACITOR
}

\author{
NIHAD DIB $^{\mathrm{a}, *, \dagger}$, QIU ZHANG ${ }^{\mathrm{b}}$ and ULRICH ROHDE ${ }^{\mathrm{c}}$ \\ ${ }^{a}$ Electrical Engineering Department, Jordan University of Science and Technology, P.O. Box 3030, \\ Irbid 22110, Jordan; ' Ansoft Corporation, Elmwood Park, NJ, USA; \\ ${ }^{\mathrm{c} S y n e r g y ~ M i c r o w a v e ~ C o r p o r a t i o n, ~ P a t e r s o n, ~ N J, ~ U S A ~}$
}

(Received 7 October 2003; In final form 17 November 2003)

\begin{abstract}
A new model for the microstrip line interdigital capacitor is proposed. This model consists of calculating the $2 N \times 2 N Y$-matrix of the $N$ coupled lines using the multiple coupled line tool. Then, this matrix is reduced to a $2 \times 2$ matrix using simple circuit theory. The capacitance at the end of each finger is taken into consideration using closed-form expression of the coplanar waveguide open-end capacitance. This model can predict the resonances that might appear around the quarter-wavelength frequency. These resonances are caused by the coupling between the fingers and exist only in capacitors with four fingers or more. Very good agreement is obtained between the results of our model and those obtained using the software HFSS and measurements.
\end{abstract}

Keywords: Capacitors; Interdigital capacitors; Microstrip capacitors; Multiple coupled lines

\section{INTRODUCTION}

Interdigital capacitors (ICAPs) are frequently used in microstrip (MS) microwave integrated circuits. The properties of this type of capacitor have been studied by many authors [1-15]. Coupled line theory was used in Refs. [1,3-5] to derive the impedance matrix of these capacitors. Many assumptions were made, some of which are: (1) only coupling between adjacent fingers is considered; (2) the number of fingers is large such that a periodic smooth structure can be assumed; (3) capacitor dimensions are much less than a quarter wavelength; and (4) capacitance at the end of each finger is neglected. An improved model in which the capacitor is divided into its basic subcomponents was very briefly described in Ref. [6]. These subcomponents included bends, T-junctions, gaps, open-ends, and uniform transmission lines. Existing computer models were used to characterize these subcomponents. Fullwave analysis and/or measurements were used in Refs. $[8,12,14,15]$ to study the MS ICAP. A measurement-based lumped-element model was given in Ref. [7]. Conformal mapping was used in Refs. $[9,11,13]$ to derive simple closed-form expressions for the capacitance of multilayered ICAPs.

\footnotetext{
* Corresponding author. Tel.: +962-7095111, ext. 22616; Fax: +962-2-7095018; E-mails: nihad@just.edu.jo

† Formerly with Ansoft Corporation, Elmwood Park, NJ.
} 
In this article, a new model for the MS ICAP is proposed and tested. In this model, all the coupling effects between the fingers are taken into account using the fast multiple coupled line (MCPL) tool in the CAD software Serenade [16]. In this tool, the $2 N \times 2 N$ admittance matrix of the $N$-fingers' section is obtained using the Spectral Domain technique, which is then reduced to $2 \times 2$ matrix using simple circuit theory. The capacitance at the end of each finger is taken into consideration using closed-form expression of the coplanar waveguide (CPW) open-end capacitance [17]. It is shown that this model can predict the resonances that might appear due to coupling when long fingers are considered. To our knowledge, these resonances occuring in ICAPs have not been reported in the literature before.

The model is briefly described in Section 2, while validation and numerical results are presented in Section 3.

\section{CAPACITOR MODEL}

Figure 1 shows the MS ICAP under invesigation. The ICAP itself consists of $N$ fingers, each of width $W$, with an overlapping distance $L_{p}$. The number of fingers $N$ could be even or odd. An odd $N$ would give different phases for $S_{11}$ and $S_{22}$, which cannot be predicted by the coupled-line theory models mentioned above. The separation distance $S$ between adjacent fingers is assumed to be constant. Similarly, the widths of all fingers are assumed to be equal (denoted by $W$ ). At the end of each finger, there is a gap with width $G$ that gives rise to an end-capacitance between each finger and the terminal strips. The terminal strips have equal lengths of $W_{\mathrm{T}}$, and are fed with MS lines of widths $W_{f 1}$ and $W_{f 2}$.

Our approach in modeling the MS ICAP can be summarized in the following steps.

1. First, the MCPL tool [16], which takes into account all the coupling effects between the fingers, is used to derive the $2 N \times 2 N$ Y-matrix of the ICAP fingers. It should be mentioned that one main advantage of using the MCPL tool, although not investigated here, is the ability to analyze an ICAP with different strip widths and/or different spacings between the fingers.

2. The second step is to reduce this $2 N \times 2 N$ Y-matrix to a $2 \times 2$ matrix. After extensive experiments, it has been found that the ICAP structure can be effectively modeled as shown in Figure 2. The capacitors model the gap capacitances that exist at the end of each finger. This capacitance can be approximately modeled as a CPW open-end capacitance $[11,13]$. The formula for the CPW open-end capacitance given in Ref. [17] is used to compute this capacitance. This formula, which was derived analytically in Ref. [17] under narrow-slot assumption, has been found to give very good results for the end-capacitance.

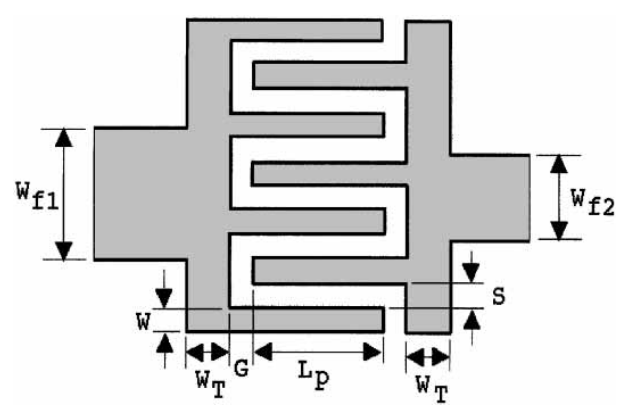

FIGURE 1 A 7-fingers MS ICAP. 


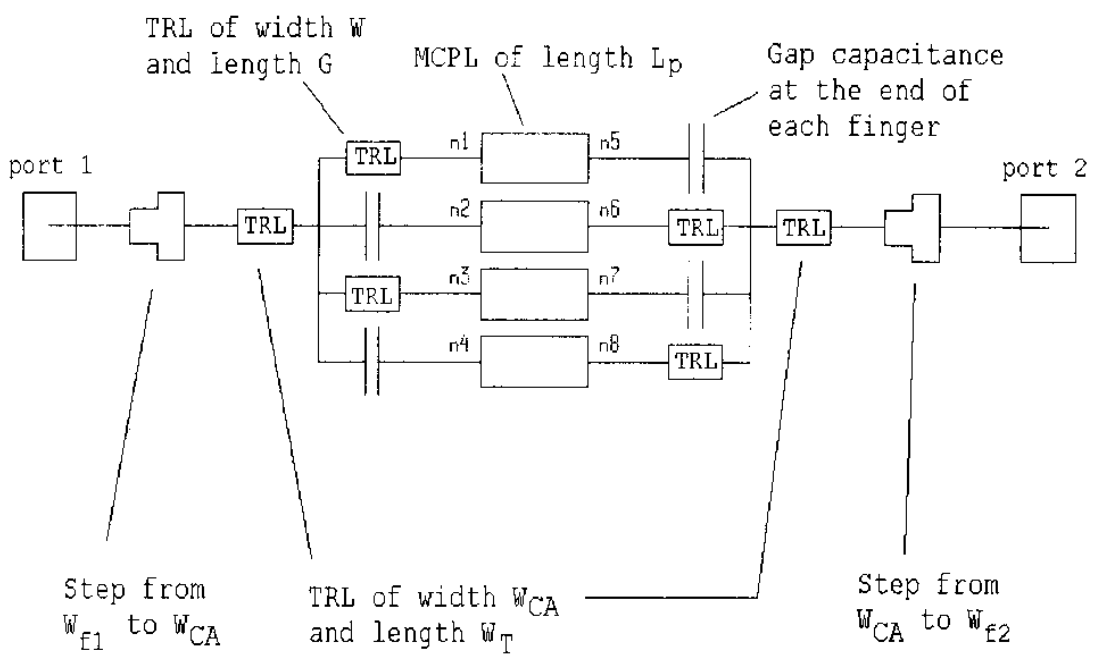

FIGURE 2 Equivalent model for a 4-fingers MS ICAP. $W_{\mathrm{CA}}$ is the total width of the capacitor.

Alternatively, for wide gaps, the simple asymptotic value for the end-capacitance given in Ref. [18] can be used. In Figure 2, the transmission line (TRL) sections, connected directly to the MCPL nodes, are used to model those small MS sections at the beginning of each finger. These strip sections are of length $G$ and width $W$. This model has proven to give very good results compared to full-wave results obtained using the HFSS software [16], as will be shown below.

3. To simplify the problem of reducing the $2 N \times 2 N$ Y-matrix to a $2 \times 2$ matrix, the small TRL sections were replaced by inductors. The inductance for each section is simply given by [19]:

$$
Z_{L}=j \omega L=j Z_{0} \beta \ell
$$

where $\ell$ is equal to the gap width $G, Z_{0}$ is the characteristic impedance of an MS line of width $W$, and $\beta$ is the phase constant. This replacement of the TRL sections by inductors is facilitated by the fact that their lengths are very small compared to the wavelength, and the fact that they have high impedance (since the finger width is small). The effect of these inductors on the response of the ICAP has been found to be very small. However, their effect becomes noticeable at high frequencies.

4. Figure 3 shows the final model of the ICAP itself. What is needed is the $2 \times 2$ Y-matrix that relates the input and output currents and voltages $\left(I_{1}^{t}, I_{2}^{t}, V_{1}^{t}\right.$, and $\left.V_{2}^{t}\right)$, given the $2 N \times 2 N$ $Y$-matrix of the MCPL section that relates the currents and voltages at the finger terminals $\left(I_{1}, \ldots, I_{2 N} ; V_{1}, \ldots, V_{2 N}\right)$. The main steps in this derivation are presented below.

From Figure 3, the following set of equations can be written:

$$
\begin{aligned}
& I_{1}=\frac{V_{1}^{t}-V_{1}}{Z_{L}}=Y_{1,1} V_{1}+Y_{1,2} V_{2}+\cdots+Y_{1,2 N} V_{2 N}, \\
& I_{2}=\left(V_{1}^{t}-V_{2}\right) Y_{c}=Y_{2,1} V_{1}+Y_{2,2} V_{2}+\cdots+Y_{2,2 N} V_{2 N}, \\
& I_{3}=\frac{V_{1}^{t}-V_{3}}{Z_{L}}=Y_{3,1} V_{1}+Y_{3,2} V_{2}+\cdots+Y_{3,2 N} V_{2 N},
\end{aligned}
$$




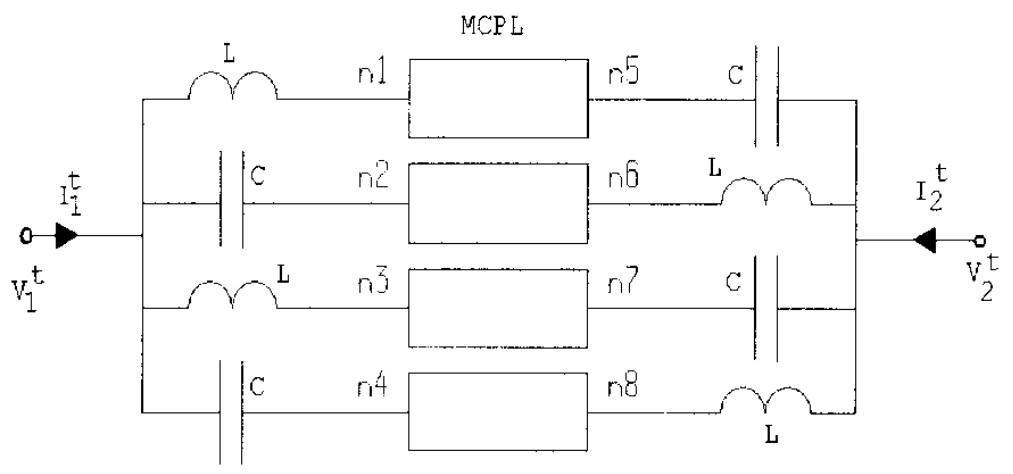

FIGURE 3 Final MCPL model for a 4-fingers ICAP.

where $Y_{c}=j \omega C$. Similar equations can be written for the rest of the MCPL nodes. The above $2 N$ equations can be written in matrix form as:

$$
[A][V]=[B]\left[V^{t}\right],
$$

where $[A]$ is a $2 N \times 2 N$ matrix whose elements are related to the elements of the MCPL $Y$-matrix, $[V]$ is a $2 N \times 1$ vector that contains the voltages at the MCPL nodes, $[B]$ is a $2 N \times 2$ matrix, and $\left[V^{t}\right]$ is a $2 \times 1$ vector. From the above equation, one can get all the voltages at the MCPL nodes as functions of $V_{1}^{t}$ and $V_{2}^{t}$ by inverting the matrix $[A]$ numerically and multiplying it by the matrix $[B]$.

Then, using the fact that

$$
\begin{aligned}
& I_{1}^{t}=I_{1}+I_{2}+\cdots+I_{N} \\
& I_{2}^{t}=I_{N+1}+I_{N+2}+\cdots+I_{2 N}
\end{aligned}
$$

one can write the following matrix equalities:

$$
\left[I^{t}\right]=[D][I]=[D][Y][V]=[D][Y][A]^{-1}[B]\left[V^{t}\right]=\left[Y^{t}\right]\left[V^{t}\right]
$$

where $[D]$ is a $2 \times 2 N$ matrix obtained from Eqs. (6) and (7), and $\left[Y^{t}\right]$ is the needed $2 \times 2$ matrix.

5. It should be emphasized that the above $2 \times 2$ Y-matrix models the ICAP itself, i.e., the fingers and the gaps at the end of the fingers. Finally, the rest of the ICAP structure could be taken into account, as explained in Ref. [6], using TRL sections, steps, bends, and T-junction elements.

\section{RESULTS}

Extensive numerical experiments have been carried out using the above model. A sample of the obtained results is presented here.

1. The first example has $N=9, W=0.4 \mathrm{~mm}, S=G=0.2 \mathrm{~mm}, L_{p}=1.4 \mathrm{~mm}, W_{\mathrm{T}}=0.4 \mathrm{~mm}$, $W_{F 1}=W_{F 2}=1.2 \mathrm{~mm}$, substrate thickness $h=1.27 \mathrm{~mm}$, and dielectric constant $\varepsilon_{r}=10.2$. The feeding lines have a $50 \Omega$ characteristic impedance. Figures 4 and 5 show the obtained scattering parameters as compared to those obtained using the full-wave finite element 


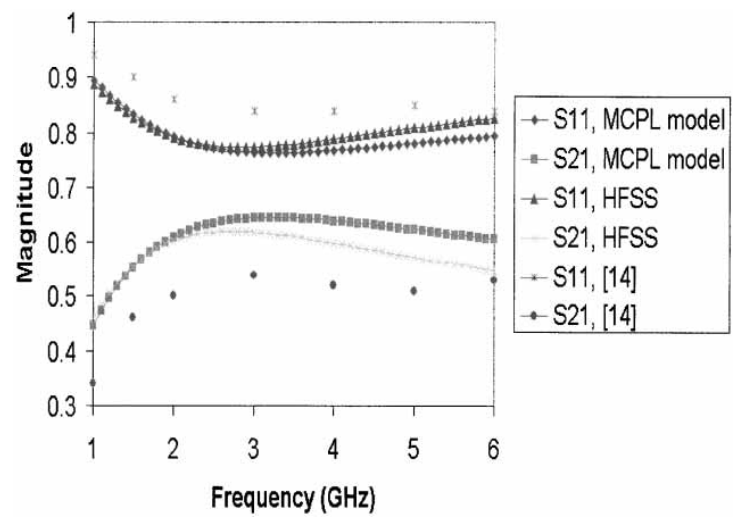

FIGURE 4 Magnitude of scattering parameters of an MS ICAP. $N=9, W=0.4 \mathrm{~mm}, S=G=0.2 \mathrm{~mm}$, $L_{p}=1.4 \mathrm{~mm}, W_{\mathrm{T}}=0.4 \mathrm{~mm}, W_{F 1}=W_{F 2}=1.2 \mathrm{~mm}$, substrate thickness $h=1.27 \mathrm{~mm}$, and dielectric constant $\varepsilon_{r}=10.2$.

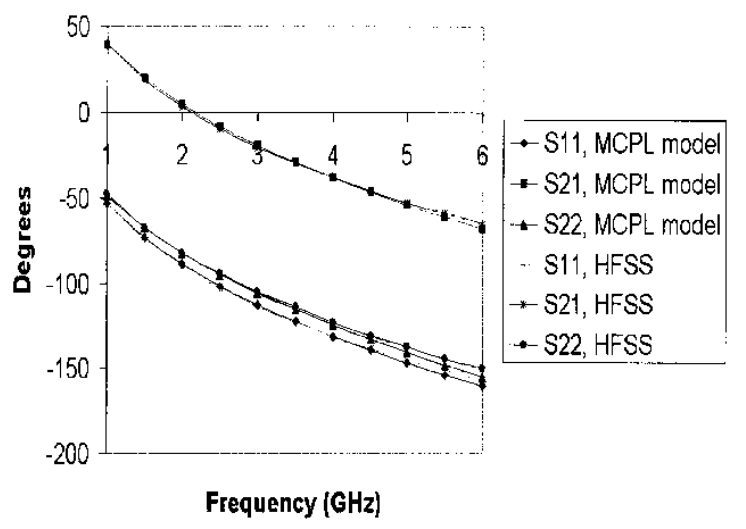

FIGURE 5 Phase of scattering parameters of an MS ICAP. Refer to Figure 4 for dimensions.

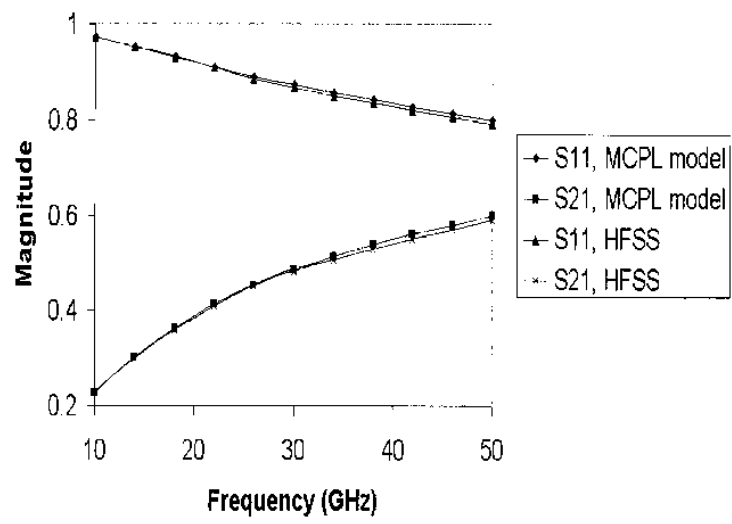

FIGURE 6 Magnitude of scattering parameters of an MS ICAP. $N=5, W=25 \mu \mathrm{m}, S=G=20 \mu \mathrm{m}, L_{p}=300 \mu \mathrm{m}$, $W_{\mathrm{T}}=50 \mu \mathrm{m}, W_{F 1}=W_{F 2}=100 \mu \mathrm{m}, h=50 \mu \mathrm{m}$, and $\varepsilon_{r}=12.9$. 


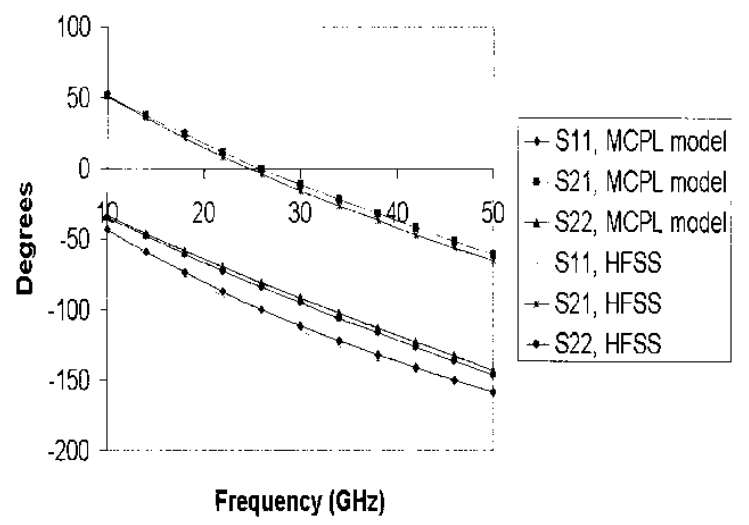

FIGURE 7 Phase of scattering parameters of an MS ICAP. Refer to Figure 6 for dimensions.

software HFSS [16]. It can be seen that good agreement is obtained, with a maximum difference of $4 \%$ for $S_{11}$, and $9 \%$ for $S_{21}$ at $6 \mathrm{GHz}$. This difference could be due to the parasitic effects inherent in the ICAP structure, which are neglected in the MCPL model. For example, in the MCPL model, the coupling between the terminal strips, and their coupling
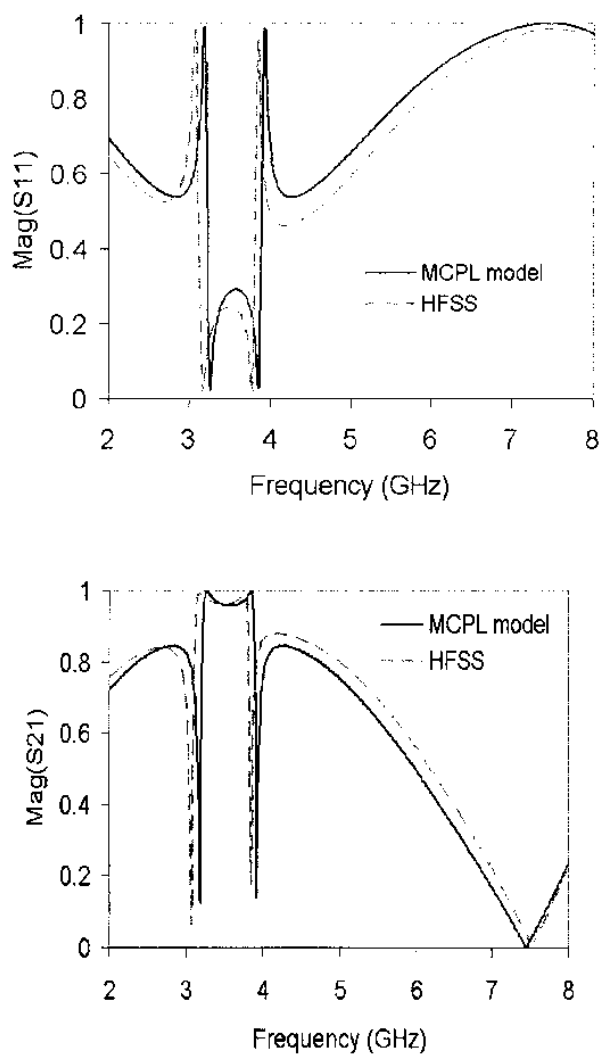

FIGURE 8 Magnitude of scattering parameters of a 4-fingers MS ICAP. $W=0.316 \mathrm{~mm}, S=0.3 \mathrm{~mm}$, $G=0.341 \mathrm{~mm}, L_{p}=13.7 \mathrm{~mm}, W_{\mathrm{T}}=0.5 \mathrm{~mm}, W_{F 1}=W_{F 2}=1.868 \mathrm{~mm}, h=0.787 \mathrm{~mm}$, and $\varepsilon_{r}=3.2$. 
to the fingers of the ICAP are neglected. Moreover, the parasitic effects of the several bends and T-junctions, existing in the ICAP structure, and radiation loss are neglected in the MCPL model. A very fine mesh had to be considered in the HFSS simulation to reach convergence. On the other hand, it took only a few seconds to obtain the results using the MCPL model in the whole frequency range (with $0.01 \mathrm{GHz}$ frequency increment). An ICAP with the same dimensions was analyzed and measured in Ref. [14] and the theoretical results are shown in Figure 4. A full-wave method of moments was used for the analysis in Ref. [14]. The cause of the difference between our results (both MCPL model and HFSS) and those from Ref. [14] is not clear. The phase of the scattering parameters was not shown in Ref. [14]. It can be seen from Figure 5 that the phase of $S_{11}$ is slightly different from the phase of $S_{22}$ since an odd number of fingers is considered.

2. The second example has $N=5, W=25 \mu \mathrm{m}, S=G=20 \mu \mathrm{m}, L_{p}=300 \mu \mathrm{m}, W_{\mathrm{T}}=50 \mu \mathrm{m}$, $W_{F 1}=W_{F 2}=100 \mu \mathrm{m}, h=50 \mu \mathrm{m}$, and $\varepsilon_{r}=12.9$. It should be noted that the characteristic impedance of the feeding lines is around $30 \Omega$, which is used as reference for the $S$-parameter calculations. Figures 6 and 7 show the results. The agreement between the MCPL model and HFSS results is very good in the whole frequency range from 10 to $50 \mathrm{GHz}$. It should be noted that although an open structure is considered in the HFSS simulation, the agreement with the MCPL model results is very good, which indicates that the radiation loss from this ICAP is very small.

3. As a third example, an MS ICAP with the following dimensions has been analyzed: $N=4, \quad W=0.316 \mathrm{~mm}, \quad S=0.3 \mathrm{~mm}, \quad G=0.341 \mathrm{~mm}, \quad L_{p}=13.7 \mathrm{~mm}, \quad W_{\mathrm{T}}=0.5 \mathrm{~mm}$,
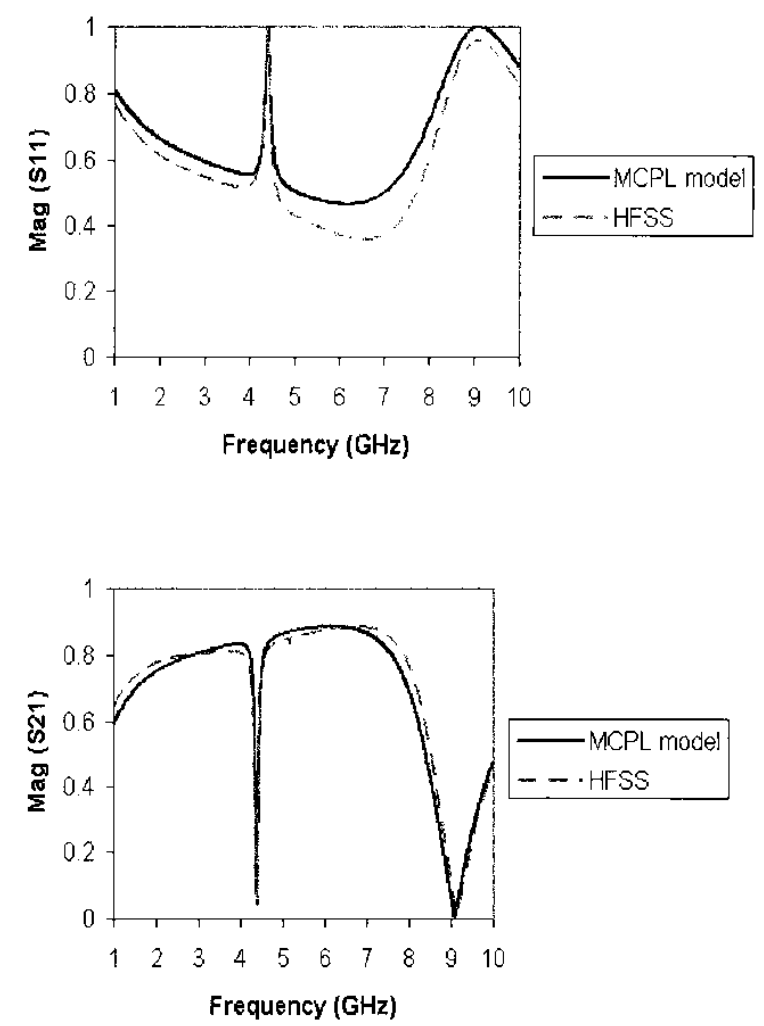

FIGURE 9 Magnitude of scattering parameters for a 5-fingers MS ICAP. $W=0.15 \mathrm{~mm}, S=0.125 \mathrm{~mm}$, $G=0.125 \mathrm{~mm}, L_{p}=6.9 \mathrm{~mm}, W_{\mathrm{T}}=0.3 \mathrm{~mm}, W_{F 1}=W_{F 2}=0.56 \mathrm{~mm}, h=0.635 \mathrm{~mm}$, and $\varepsilon_{r}=10.2$. 
$W_{F 1}=W_{F 2}=1.868 \mathrm{~mm}, h=0.787 \mathrm{~mm}$, and $\varepsilon_{r}=3.2$. Figure 8 shows the results obtained using the MCPL model as compared to HFSS simulations. It is very interesting to notice the resonances appearing between 3 and $4 \mathrm{GHz}$. In practice, an ICAP is usually used at frequencies well below the first quarter-wavelength frequency, i.e., in the region where it behaves as a capacitor with negligible parasitics.

It was found that a 3-fingers MS ICAP with the same dimensions did not show these resonances, while they continue to appear for $N$ larger than 4 fingers. Moreover, the number of these resonances was related to the number of fingers, as shown in the next example. After extensive simulations, it was concluded that these resonances are due to the coupling between the fingers. They occur around the frequencies at which the length of the ICAP is an odd multiple of quarter wavelength. These resonances are similar to those occuring in coupled lines bandpass filters. To our knowledge, such resonances occuring in interdigital capacitors have not been shown before in the literature.

4. Figure 9 shows the scattering parameters for an MS ICAP with the following dimensions: $N=5, W=0.15 \mathrm{~mm}, S=0.125 \mathrm{~mm}, G=0.125 \mathrm{~mm}, L_{p}=6.9 \mathrm{~mm}, W_{\mathrm{T}}=0.3 \mathrm{~mm}$, $W_{F 1}=W_{F 2}=0.56 \mathrm{~mm}, h=0.635 \mathrm{~mm}$, and $\varepsilon_{r}=10.2$. It can be noticed that a single resonance appears around $4.5 \mathrm{GHz}$ in both the MCPL model and HFSS simulations. Again, the agreement is very good, which validates the proposed MCPL model.

5. As a last example, Figure 10 shows the scattering parameters for an MS ICAP with the following dimensions: $N=4, W=0.5 \mathrm{~mm}, S=0.5 \mathrm{~mm}, G=0.5 \mathrm{~mm}, L_{p}=14 \mathrm{~mm}$,
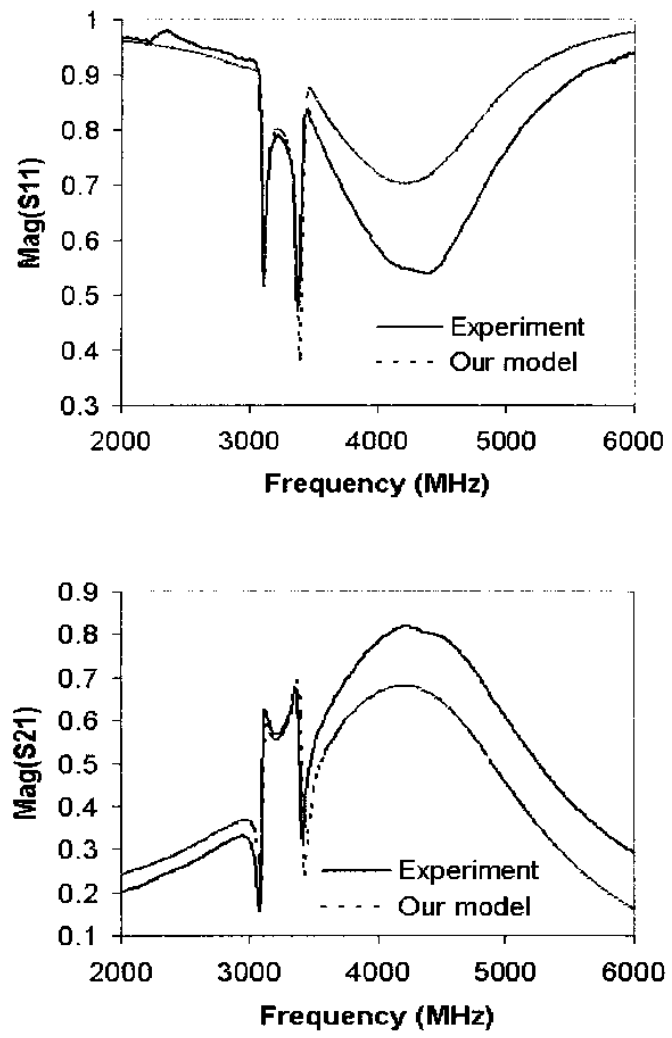

FIGURE 10 Magnitude of scattering parameters for a 4-fingers MS ICAP. $N=4, W=0.5 \mathrm{~mm}, S=0.5 \mathrm{~mm}$, $G=0.5 \mathrm{~mm}, L_{p}=14 \mathrm{~mm}, W_{\mathrm{T}}=1 \mathrm{~mm}, W_{F 1}=W_{F 2}=2.1 \mathrm{~mm}, h=0.508 \mathrm{~mm}$, and $\varepsilon_{r}=3.38$ 
$W_{\mathrm{T}}=1 \mathrm{~mm}, W_{F 1}=W_{F 2}=2.1 \mathrm{~mm}, h=0.508 \mathrm{~mm}$, and $\varepsilon_{r}=3.38$. Experimental results are also shown in the figure. The circuit was fabricated on Rogers $4003 \mathrm{C}$ substrate (with $34 \mu \mathrm{m}$ copper thickness) using the LPKF ProtoMat 91s/VS mill/drill unit, which is usually used to produce prototype PCBs. Measurements were performed using an HP 8510 Network Analyzer, along with TRL calibration. It can be seen that both the MCPL model and experiments predict the resonances at the same frequencies. The differences can be attributed to the finite size ground plane used in the measurements, calibration errors, connectors' effects, and the other parasitic effects mentioned above in the first example.

\section{CONCLUSIONS}

A new model for the MS ICAP has been developed. This model is based on the use of the MCPL programs to obtain the $Y$-matrix of the $N$ coupled fingers. Then, it is reduced to a $2 \times 2$ admittance matrix using simple network theory. This new model has been verified by comparison to full-wave results obtained using HFSS. It has been shown that a band-pass filterlike effect can exist in interdigital capacitors with four fingers or more. This effect has been validated by HFSS simulations and experiments.

\section{References}

[1] Alley, G. (1970). Interdigital capacitors and their application to lumped-element microwave integrated circuits. IEEE Trans. on MTT, 1028-1033.

[2] Lacombe, D. and Cohen, J. (1972). Octave-band microstrip DC blocks. IEEE Trans. on MTT, 555-556.

[3] Hobdell, J. (1979). Optimization of interdigital capacitors. IEEE Trans. on MTT, 788-791.

[4] Esfandiari, R., Maki, D. and Siracusa, M. (1983). Design of interdigital capacitors and their application to GaAs monolithic filters. IEEE Trans. on MTT, 57-64.

[5] She, X. and Chow, Y. (1986). Interdigital microstrip capacitor as a four-port network. IEE Proc., 133, Pt. H, (3), 191-197.

[6] Pettenpaul, E., Kapusta, H., Weisgerber, A., Mampe, H., Luginsland, J. and Wolff, I. (1988). CAD models of lumped elements on GaAs up to $18 \mathrm{GHz}$. IEEE Trans. on MTT, 294-304.

[7] Sadhir, V., Bahl, I. and Willems, D. (1994). CAD compatible accurate models of microwave passive elements for MMIC applications. Int. J. of Microwave and Millimeter-Wave Computer-Aided Engineering, 4(2), 148-162.

[8] Bandler, J., Biernacki, R., Chen, S., Swanson, D. and Ye, S. (1994). Microstrip filter design using direct EM field simulation. IEEE Trans. on MTT, 1353-1359.

[9] Wu, H., Zhang, Z., Barnes, F., Jackson, C., Kain, A. and Cuchiaro, J. (1994). Voltage tunable capacitors using high temperature superconductors and ferroelectrics. IEEE Trans. on Applied Superconductivity, 4(3), 156-160.

[10] David, G., Bussek, P., Auer, U., Tegude, F. and Jager, D. (1995). Electro-optic probing of RF signals in submicrometer MMIC devices. Electronics Letters, 31(25), 2188-2189.

[11] Gevorgian, S., Martinsson, T., Linner, P. and Kolberg, E. (1996). CAD models for multilayered substrate interdigital capacitors. IEEE Trans. on MTT, 896-904.

[12] Coen, G., de Zutter, D. and Fache, N. (1996). Automatic derivation of equivalent circuits for general microstrip interconnection discontinuities. IEEE Trans. on MTT, 1010-1016.

[13] Gevorgian, S., Carlsson, E., Rudner, S., Werulund, L., Wang, X. and Helmersson, U. (1996). Modelling of thinfilm HTS/Ferroelectric interdigital capacitors. IEE Proc. Microw. Antennas Propag., 143(5), 397-401.

[14] Zhu, L. and Wu, K. (2000). Accurate circuit model of interdigital capacitor and its application to design of new quasi-lumped miniaturized filters with suppression of harmonic resonance. IEEE Trans. on MTT, 347-356.

[15] Gharsallah, A., Gharbi, A., Desclos, L. and Baudrand, H. (2002). Analysis of interdigital capacitors and quasilumped miniaturized filters using iterative method. Int. J. of Numerical Modelling: Electronic Networks, Devices and Fields, 15, 169-179.

[16] Ansoft Corporation, Pittsburgh, USA 15219.

[17] Mao, M., Wu, R., Chen, C. and Lin, C. (1994). Characterization of CPW open end capacitance theory and experiment. IEEE Trans. on MTT, 1016-1024.

[18] Beilenhoff, K., Klingbeil, H., Heinrich, W. and Hartuagel, H. (1993). Open and short circuits in coplanar MMICs. IEEE Trans. on MTT, 1534-1537.

[19] Pozar, D. (1998). Microwave Engineering, 2nd ed., John Wiley \& Sons, Inc. 

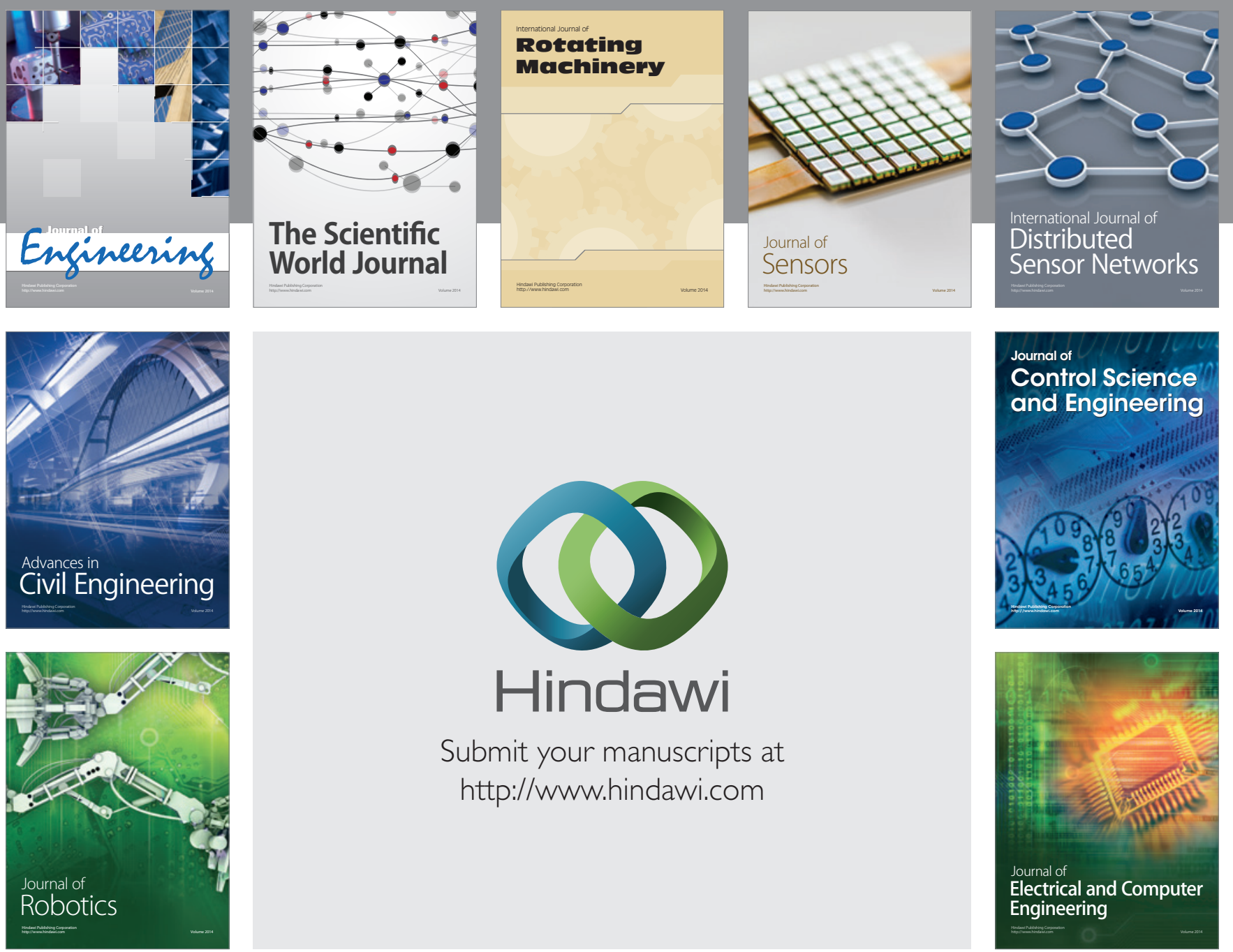

Submit your manuscripts at

http://www.hindawi.com
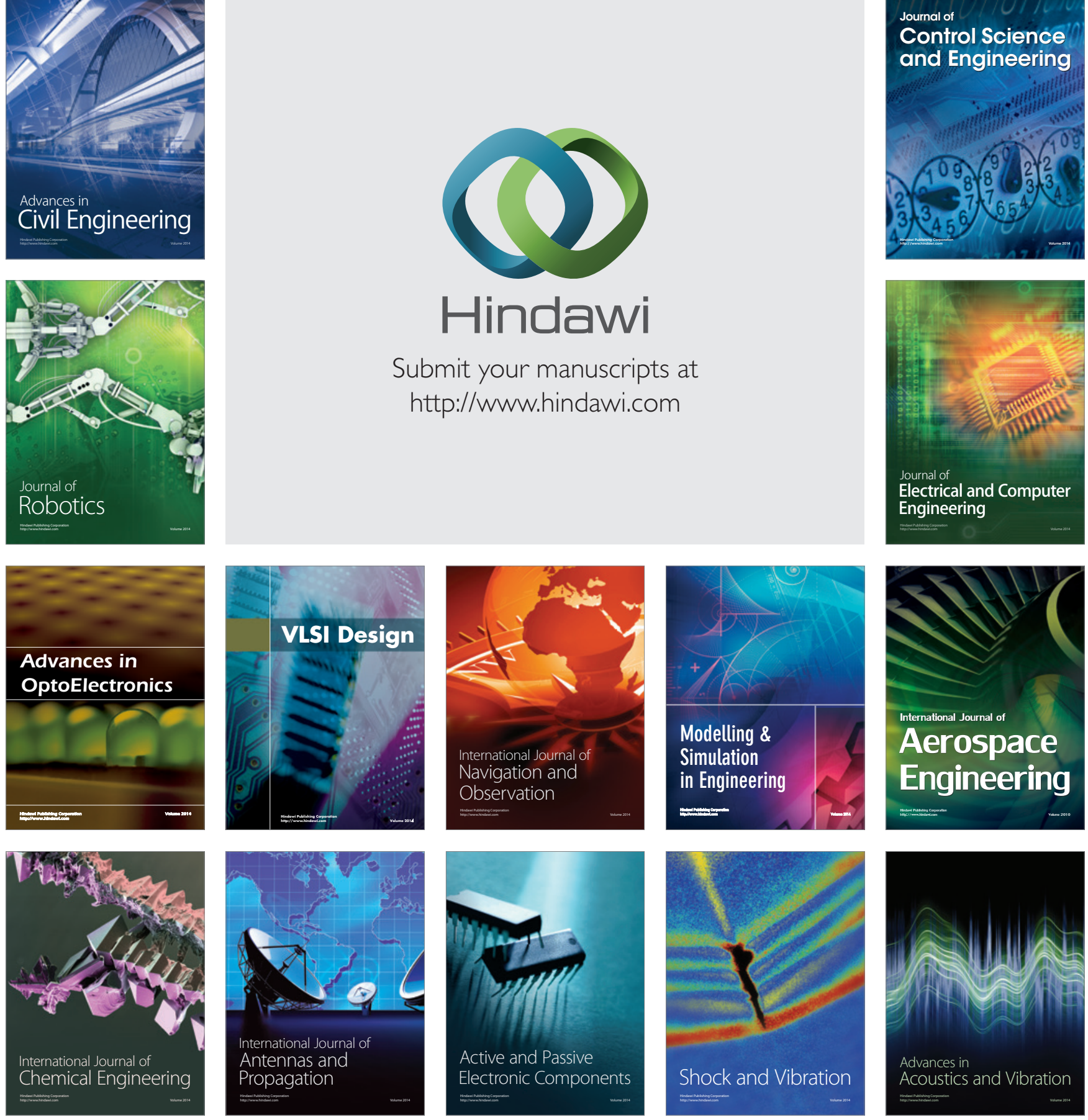